\title{
Retardation Effects in Valence - EELS Spectra
}

\author{
M. Stöger-Pollach, ${ }^{*}$ C. Hébert, ${ }^{* *}$ P. Schattschneider,** and A. Laister** \\ * University Service Center for TEM, Vienna University of Technology, A-1040 Vienna, Austria \\ ** Institute for Solid State Physics, Vienna University of Technology, A-1040 Vienna, Austria
}

Čerenkov losses in EELS remain an often neglected problem in spite of the comprehensive description of the phenomenon by Kröger [1] and Chen et al. [2] more than 30 years ago. Modern instruments equipped with monochromators and high-end energy filters or spectrometers allow nowadays determination of band gaps, the joint density of states, and the dielectric function with unprecedented accuracy. But especially in semiconductors and insulators, where $\mathscr{R}_{e}(\varepsilon)$ is relatively large and $\mathscr{C}_{m}(\varepsilon)$ is small (or zero), the condition for Čerenkov radiation $v>c_{0} / n$ is often fulfilled (where $\mathrm{n}$ is the refractive index, $v$ is the velocity of the electron and $c_{0}$ the speed of light). The excitation of Čerenkov radiation leads to a retardation of the swift electron and therefore to a signal in the bandgap and interband transition region of the EELS spectrum. An understanding of relativistic effects in the very low energy loss range is therefore at stake because it is this effect which prevents a simple interpretation of valence EELS (VEELS) spectra of semiconductors or insulators in terms of band gaps.

It can be shown that the experimental loss spectrum can be decomposed into a longitudinal (Coulombic) and a transverse (relativistic) part, the latter describing Čerenkov losses. Experimental results on a number of semiconductors, including angle resolved measurements compare favourably with a relativistic calculation. The importance of surfaces for radiation losses is demonstrated. For thin sample the surface waves are not isolated on their particular surfaces but coupled. The possibility for such a coupling decreases with increasing sample thickness.

Figure 1 shows a draft simulation of the Si low loss spectrum including only relativistic volume losses (full line) and inclding the surface losses for a $220 \mathrm{~nm}$ thick sample (dashed line) at an acceleration voltage of $200 \mathrm{kV}$. If surface losses are taken into account, the Begrenzungseffect leads to a damping of the volume plason, because oszillation strength is shifted to the surface loss.

Figure 2 compares several low loss spectra recorded at different thickness with simulated ones. The collection angles in the experiment and in the simulation were $0.2 \mathrm{mrad}$ and $0.1 \mathrm{mrad}$, respectively. It is remarkable that the signal onset - which might be interpreted by laymen as "bandgap" - shifts to lower energies with increasing sample thickness. This relativistic effect is due to the weakening of surface coupling.

When measuring the bandgap of semiconductors in EELS one has to take care that no Čerenkov losses influence the spectrum. If this is the case or not can easily be estimated in table 1, where the refractive indices above which Čerenkov contributions appear are linked with the operation voltage of conventional TEMs. If Čerenkov losses appear, a proper determination of the bandgap by simply reading the signal onset is impossible. Also Kramers-Kronig analysis leads to erroneous results of optical properties. 
References

[1] E. Kröger, Z. Phys. 216 (1968), 115.

[2] C, H. Chen, J. Silcox and R. Vincent, Phys. Rev. B 12 (1975) 64.

[3] Financial support was given by the European Project METEOR, Contr. Nbr. ENK5 - CT $2001-00543$

TABLE 1. Critical refractive indices

Acceleration voltage Refractive index

\begin{tabular}{ll}
$100 \mathrm{kV}$ & 1.8242 \\
$200 \mathrm{kV}$ & 1.4381 \\
$300 \mathrm{kV}$ & 1.2878 \\
\hline
\end{tabular}

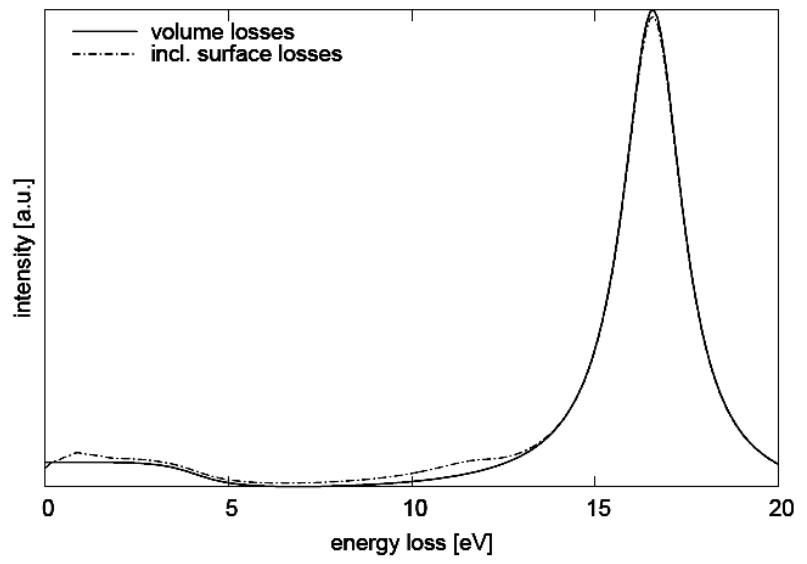

FIG. 1. Simulation of retardation effects in VEELS: volume losses (full line) and surface losses (dot-dashed line) under consideration of the surfaces for a $220 \mathrm{~nm}$ thick Si sample for $200 \mathrm{keV}$ electrons.
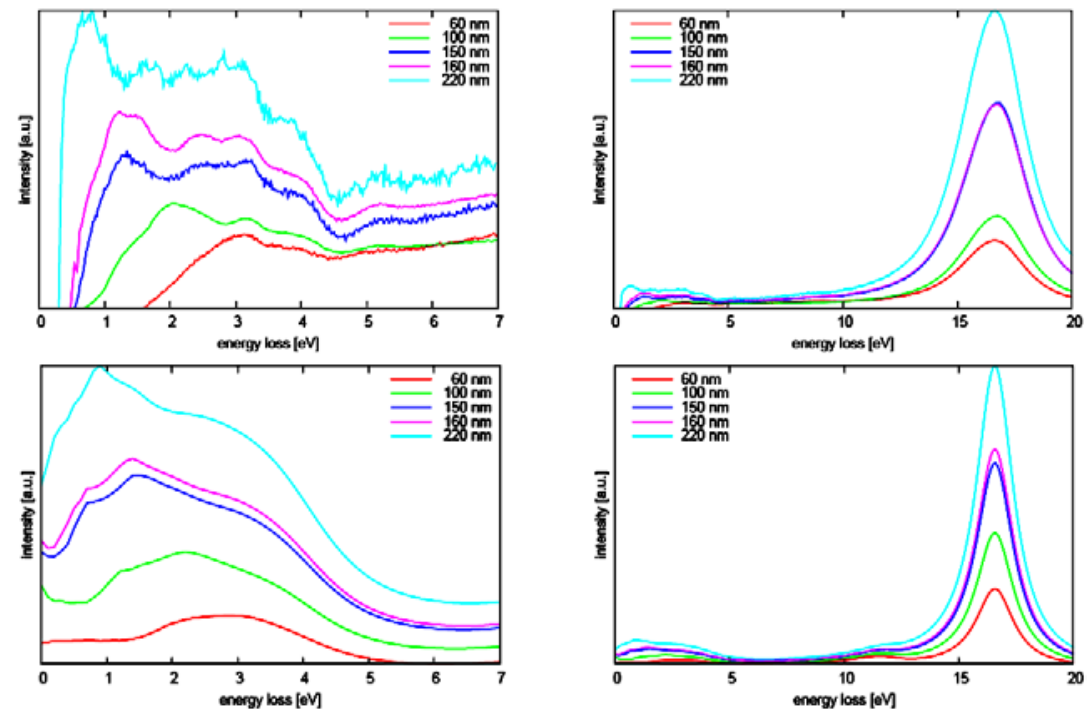

FIG. 2. Experiment (top) and simulation (bottom) of of VEELS of Si including retardation, for various thicknesses for $200 \mathrm{keV}$ electrons. 